\title{
Coating $\mathrm{ZnO}$ nanoparticle films with DNA nanolayers for enhancing the electron extracting properties and
}

\author{
performance of polymer solar cells
}

\author{
Janardan Dagar, ${ }^{1}$ Guido Scavia ${ }^{2}$ Manuela Scarselli, ${ }^{3}$ Silvia Destri, ${ }^{2}$ Maurizio De \\ Crescenzi, ${ }^{3}$ Thomas M. Brown' ${ }^{9} *$ \\ ${ }^{1}$ CHOSE (Centre for Hybrid and Organic Solar Energy), Department of Electronic Engineering, \\ University of Rome Tor Vergata, Via del Politecnico 1, 00133 Rome, Italy \\ ${ }^{2}$ CNR - ISMAC (Istituto per lo Studio delle Macromolecole) via Corti 12, 20133 Milan Italy \\ ${ }^{3}$ Department of Physics, University of Rome Tor Vergata, Via della Ricerca Scientifica 1, 00133 Rome, \\ Italy
}

Email: *thomas.brown@uniroma2.it

\section{AUTHOR INFORMATION}

\section{Corresponding Author}

*thomas.brown@uniroma2.it 


\section{ABSTRACT}

Here we present for the first time polymer solar cells that incorporate biological material that show state of the art efficiencies in excess of $8 \%$. The performance of inverted polymer solar cells was improved significantly after deposition of $\mathrm{ZnO}$ nanoparticles (ZnO-NPs) together with a thin deoxyribonucleic acid nanolayer and used as electron extraction layer (EEL). The ZnO-NPs/DNA double layers improved the rectifying ratio, shunt resistance of the cells as well as lowering the work function of the electron-collecting contact. Importantly, the ZnO-NPs/DNA bilayer enhanced the power conversion efficiency of cells considerably compared to cells with EELs made of only DNA (improvement of 56\% in relative terms) or only ZnO-NPs (improvement of $19 \%$ in relative terms) reaching a best power conversion efficiency of $8.5 \%$. The $\mathrm{ZnO}-\mathrm{NPs} / \mathrm{DNA}$ double layers cells also outperformed ones made with one of the most efficient previous synthetic composite EELs (i.e. $\mathrm{ZnO} / \mathrm{PEIE}($ poly(ethyleneimine)-ethoxylated)). Since all fabrication procedures were carried out at low $\left(<150{ }^{\circ} \mathrm{C}\right)$ or room temperature, we have applied the findings to flexible substrates as well as on glass obtaining a high PCE of 7.2\%. The solar cells with the biological/metal-oxide composite EELs also delivered an improvement in the stability ( $20 \%$ in relative term) compared to that with $\mathrm{ZnO}-\mathrm{NPs}$ only. All these findings show that natural materials, in this case DNA, the premium biological material, can be incorporated in organic semiconductor devices in tandem with inorganic devices delivering uncompromising levels of performance as well as significant improvements.

\section{KEYWORDS}

electron extraction layer, DNA nanolayer, ZnO-NPs/DNA double layers, work function, polymer solar cell 


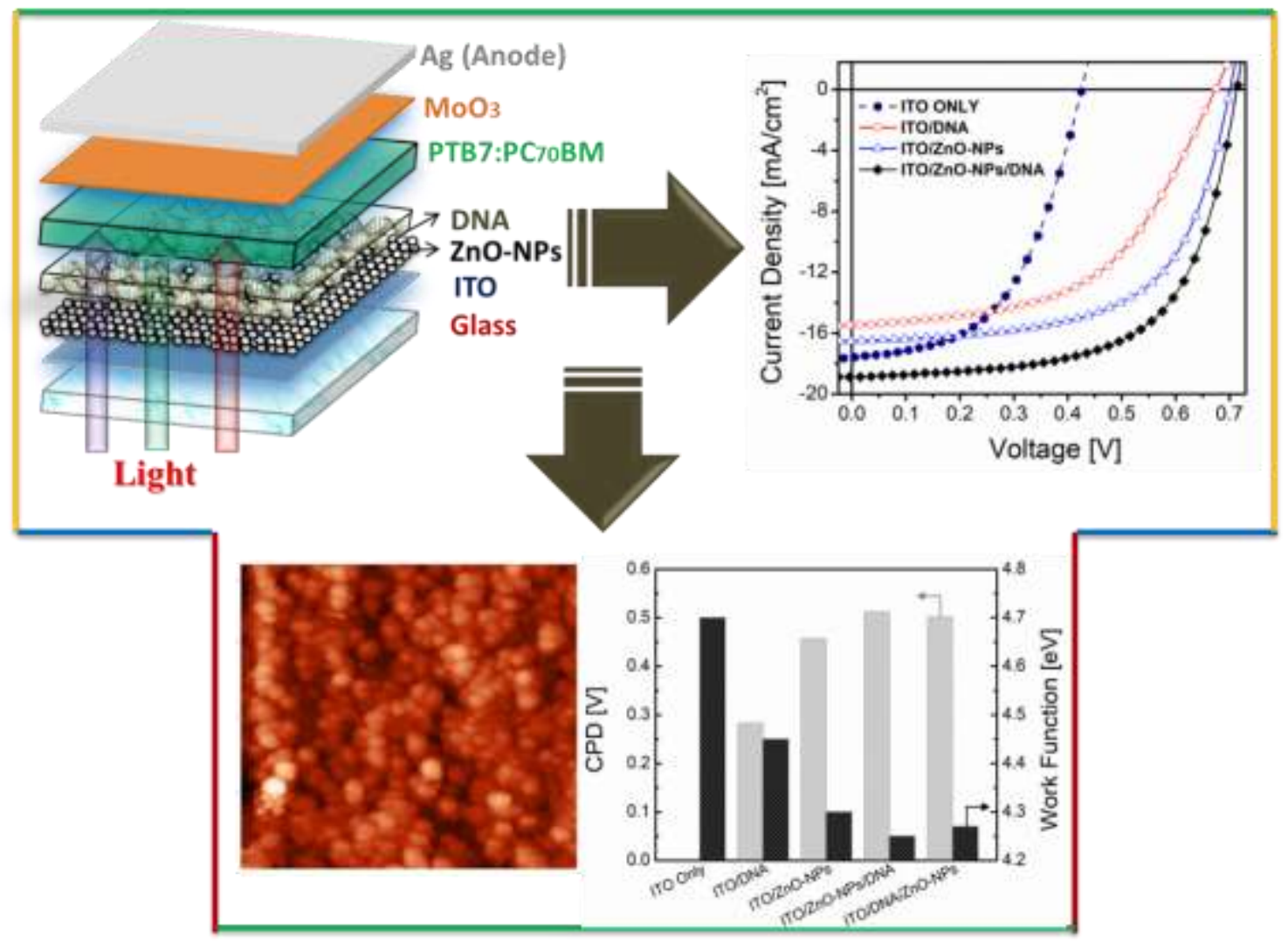




\section{Introduction}

Numerous efforts have been focused on improving the performance of polymer solar cells ${ }^{1-3}$. Many have been centered on of the identification of effective electron transport/extraction layers (EELs) of different types ${ }^{4}$ including metals, metal oxides, fluorides, caesium carbonate, fullerene derivatives $^{5}$, conjugated polyelectrolytes poly(ethyleneimine) (PEI) or poly(ethyleneimine)ethoxylated (PEIE), ${ }^{6}{ }^{7-9}$. DNA and its derivatives have also been recently ${ }^{10}$ incorporated in solar cells ${ }^{10-12}$ as single layer EELs ${ }^{11}$. DNA has also already been used to improve the performance of other organic semiconductor devices such as organic light emitting diodes (OLEDs) ${ }^{13,14}$, and organic field effect transistors (OFETs $)^{15,16}$. One of the most commonly used EELs in the inverted architecture (i.e. that with the ETL deposited over the bottom transparent contact) is that made of $\mathrm{ZnO}$, due to its high optical transparency, high electron mobility and low temperature processability ${ }^{17}$. $\mathrm{ZnO}$ is often deposited in the form of nanoparticles (NPs) delivering an effective EEL at low temperature. Nevertheless, the performance of cells may suffer from surface defects, found at the interface of $\mathrm{ZnO}-\mathrm{NPs}$ and adjacent layers, as well ITO/ZnO-NPs possessing an electron affinity which is higher ${ }^{18}$ than that of the $\mathrm{PC}_{70} \mathrm{BM}$ acceptor molecule whose LUMO lies $\sim 4.0-4.3 \mathrm{eV}$ from the vacuum level ${ }^{6,19}$. To fix these problems and enhance further the performance of solar cells, bilayer cathodes have been developed ${ }^{6}$ Here for the first time, we introduced 1-6nm thick DNA nanolayer over the ZnO-NPs layer to develop ZnO-NPs/DNA composite EELs to enhance the performance of polymer solar cells compared to EELs made of only DNA (improvement of $56 \%$ in relative terms) or only $\mathrm{ZnO}-\mathrm{NPs}$ (improvement of $19 \%$ in relative terms) reaching state of the art power conversion efficiencies (PCEs) of $8.3 \%$ and a best of $8.5 \%$. Low temperature and easy fabrication processes enabled us to transfer all layers from glass to flexible 
substrates successfully. Furthermore, we note that both ZnO-NPs and the DNA salt we used here were dissolved in an ecofreindly solvent mixture of water and ethanol.

The structure of our cell was ITO/ZnO-NPs/DNA/PTB7:PC ${ }_{70} \mathrm{BM} / \mathrm{MoO}_{3} / \mathrm{Ag}$ as shown in Fig. 1(a). The active blend consisted of PTB7 [Poly[[4,8-bis[(2-ethylhexyl)oxy]benzo[1,2-b:4,5b']dithiophene-2,6-diyl][3-fluoro-2-[(2ethylhexyl)carbonyl]thieno[3,4-b]thiophenediyl]]] as donor polymer and $\mathrm{PC}_{70} \mathrm{BM}\left[[6,6]\right.$-Phenyl-C70-butyric acid methyl ester] as acceptor ${ }^{8,19,20}$. The hole extraction layer consisted of $\mathrm{MoO}_{3}$ layer $^{20}, 21$. We used $\mathrm{ZnO}$-NPs dispersion which is commercially available ${ }^{22}$. The ZnO-NPs layer was deposited by spin coating from a dispersion of nanoparticles of average particle size $\leq 40 \mathrm{~nm}$ in water and ethanol (see experimental section), over pre-cleaned ITO/Glass or PET substrates, ${ }^{43}, 24$. In addition, we introduced over the $\mathrm{ZnO}$-NPs layer one of DNA in its "natural" 25 fibre-like salt with $\mathrm{Na}$, with the phosphate backbone being negatively charged with balancing $\mathrm{Na}^{+}\left(\right.$or H$\left.^{+}\right)$for neutrality ${ }^{26}$, resulting from the isolation ${ }^{27}$ from salmon fish, which is soluble in methanol and water ${ }^{11,28-29}$. DNA enables the fabrication of solar cells at room temperature ${ }^{11}$. 


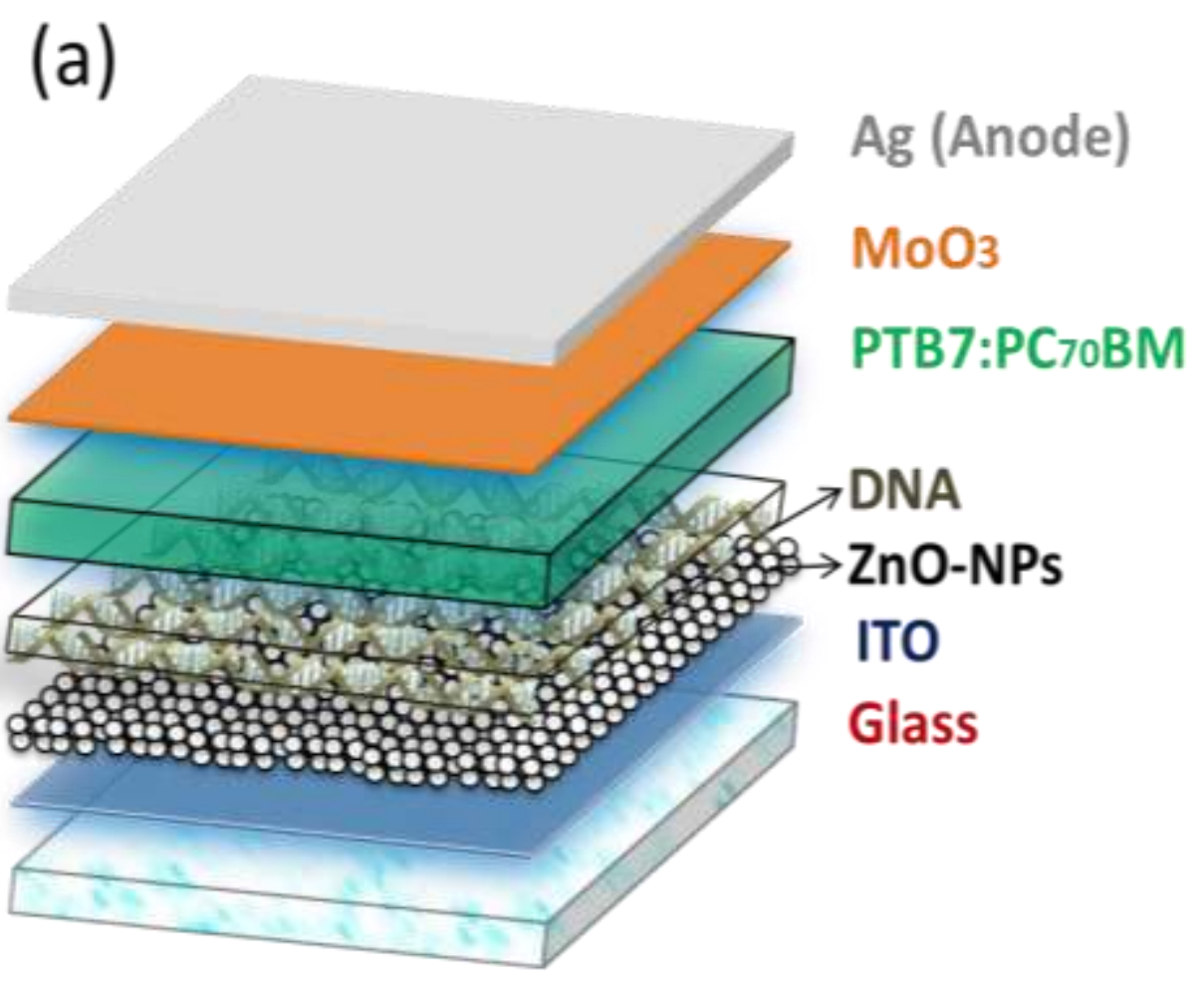

(b)

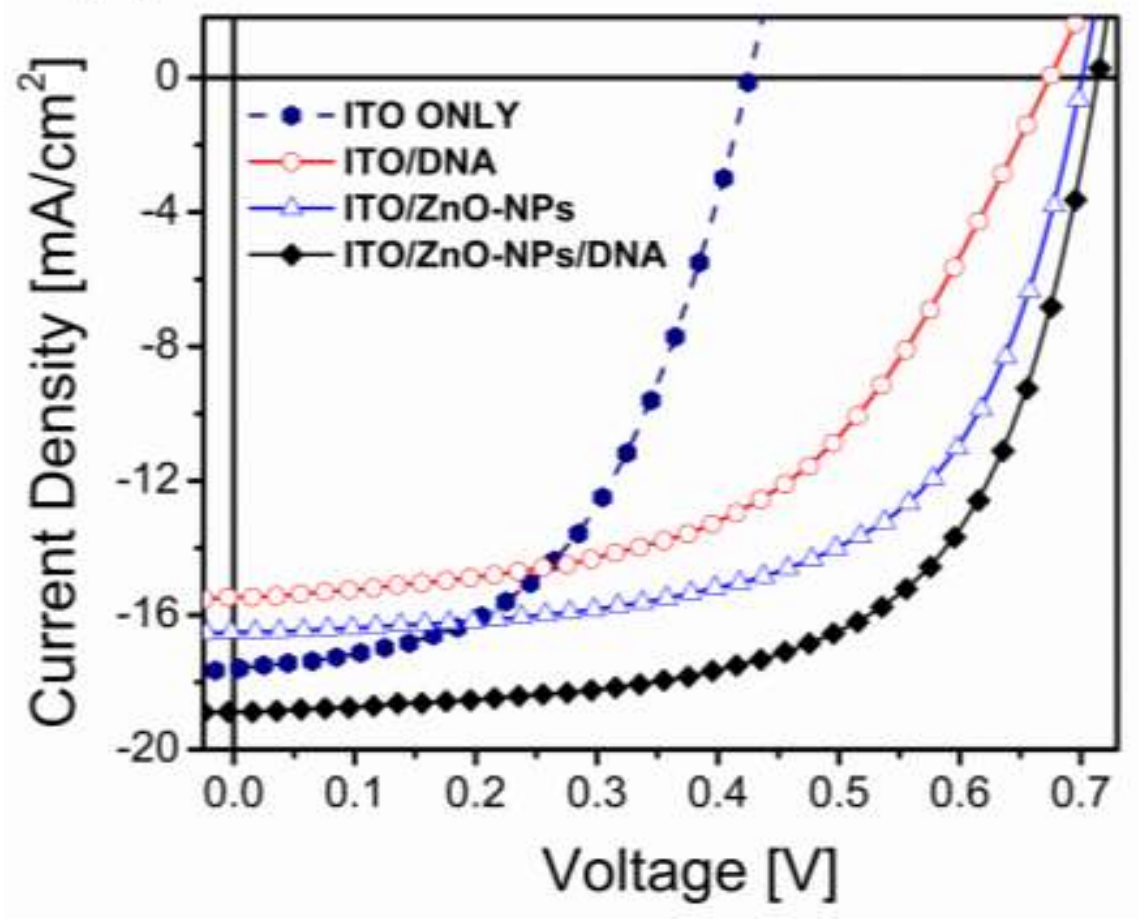




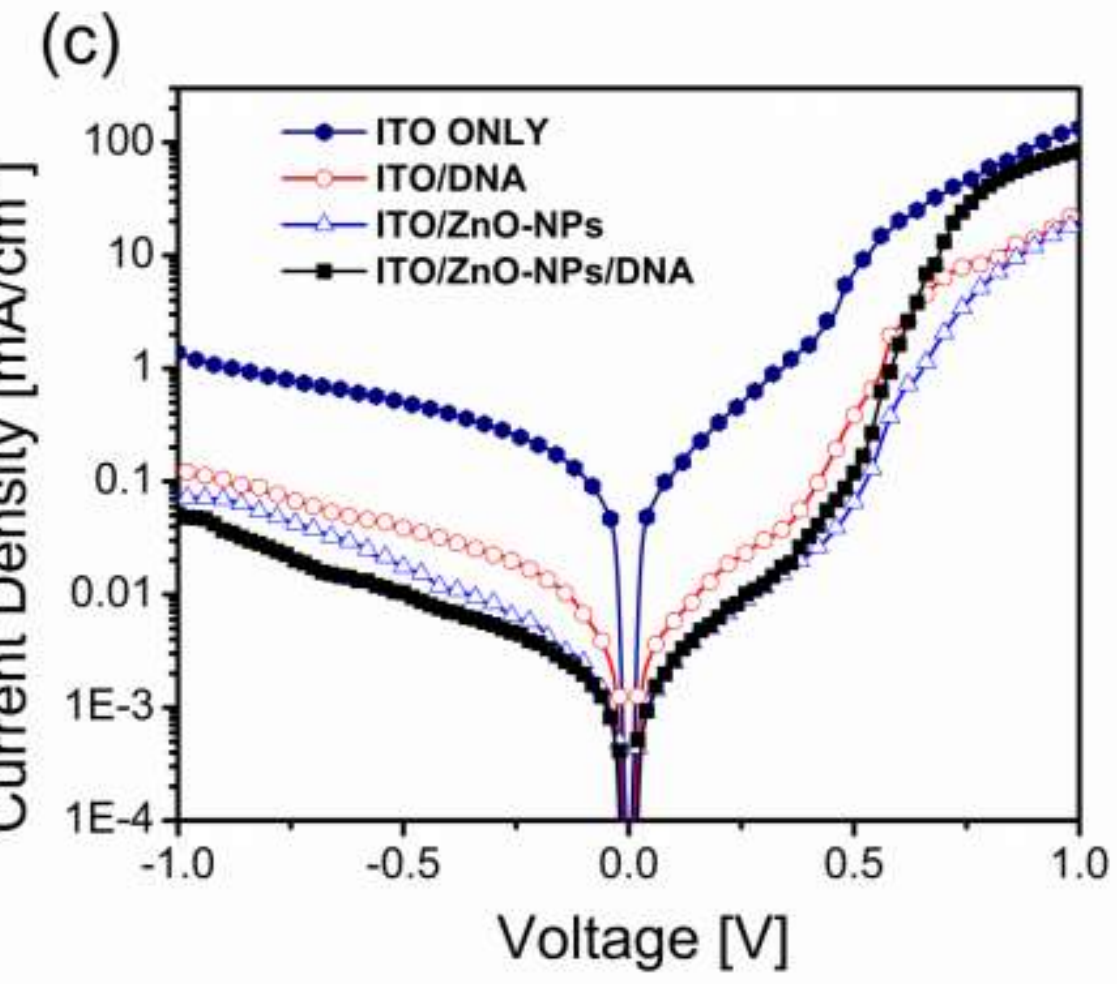

Fig.1 - (a) Schematics showing the ITO/ZnO-NPs/DNA/PTB7:PC $70 B M / M o O_{3} / A g$ inverted device architecture of polymer solar cell; (b) Best current density-voltage curves of PTB7:PC70BM solar cell devices fabricated using various interlayers including DNA (red open circle), ZnO-NPs (blue open triangle) and DNA-coated ZnO-NPs bilayer (black solid diamond) under AM1.5G, 1000 $\mathrm{W} / \mathrm{m}^{2}$ irradiation; (c) current density-voltage curves in the dark. Also reported are the JV curves of the cells with no interlayer (ITO Only, blue solid hexagon).

The current density - voltage $(J-V)$ characteristics of the best cell for inverted PTB7:PC ${ }_{70} \mathrm{BM}$ solar cells with different interlayers including DNA, ZnO-NPs, and DNA coated ZnO-NPs, measured under AM1.5G, 1000W/m² solar illumination are shown in Fig.1(b) along with those JV characteristics without any interlayer ("ITO only"). The average values of PV parameters, including short circuit current $\left(\mathrm{J}_{\mathrm{SC}}\right)$, open circuit voltage $\left(\mathrm{V}_{\mathrm{OC}}\right)$, Fill Factor $(\mathrm{FF})$ and PCE (power conversion efficiency) have been summarized in Table 1 and Fig. S1. The device with only DNA 
layer gives a PCE $=5.3 \%$ improving the PCE considerably compared to the ITO-only case (PCE $=3.46 \%)$. ZnO-NPs interlayer delivers a cell with an even better PCE $=7.0 \%$. It is when combining both $\mathrm{ZnO}-\mathrm{NPs}$ and DNA in ZnO-NPs/DNA composite bilayer EEL that leads to the most dramatic enhancement yielding a high average value of $8.3 \%$ and a maximum of $8.45 \%$ power conversion efficiency. In addition to these interlayers, we note that inverting the order of deposition of DNA/ZnO-NPs bilayers also leads to better efficiency compared to the single layer only (either DNA or ZnO-NPs): cells with DNA covered by ZnO-NPs (i.e. ITO/DNA/ZnO-NPs EELs) deliver a maximum PCE of $8.2 \%$. We have fabricated the same solar cell architecture, inserting DNA over $\mathrm{ZnO}-\mathrm{NPs}$ layer with 12 different cells and obtained consistent results. The external quantum efficiency (EQE) spectra of the solar cell devices are displayed in Fig. S2, with the highest peak in the $80 \%$ to $90 \%$ range, yielding consistent results with the Jsc, confirmed by calculating integrated Jsc value.

Fig.1(c) shows the dark J-V curves for the same cells. The highest On/Off current ratio (from $+1 \mathrm{~V} /-1 \mathrm{~V}$ ) is $1.6 \times 10^{3}$ belongs indeed to the $\mathrm{ZnO}-\mathrm{NPs} / \mathrm{DNA}$ bilayer which is significantly greater even than that of the cell with the common $\mathrm{ZnO}-\mathrm{NPs}$ only EEL $\left(2.7 \times 10^{2}\right)$. The ITO/ZnONPs/DNA bilayer cell not only exhibits the highest rectification behavior but also the lowest reverse current of all cells confirming the high quality of the electron extracting electrode. We have also fabricated solar cells with ITO/PEIE and ITO/ZnO-NPs/PEIE electron-extracting contacts in the same batch of experiments for comparison. Table 1 shows that the ITO/ZnONPs/DNA EEL is the best performing EEL of the lot. 
Table 1 - Averages of the PV parameters of PTB7:PC $70 B M$ based inverted polymer solar cells made with different interlayers over four different samples: ITO only, ITO/DNA, ITO/PEIE, ITO/ZnO-NPs, ITO/ZnO-NPS/PEIE, ITO/DNA/ZnO-NPs and DNA coated ZnO-NPs bilayer on glass and PET substrates.

\begin{tabular}{ccccc}
\hline $\begin{array}{c}\text { Electron Transport } \\
\text { Interlayers }\end{array}$ & $\begin{array}{c}\text { JsC } \\
{[\mathbf{m A} / \mathbf{c m} 2]}\end{array}$ & $\begin{array}{c}\text { Voc } \\
{[\mathbf{V}]}\end{array}$ & $\begin{array}{c}\text { FF } \\
{[\%]}\end{array}$ & $\begin{array}{c}\text { PCE } \\
{[\%]}\end{array}$ \\
\hline ITO Only & $17.63 \pm 0.03$ & $0.392 \pm 0.031$ & $50.06 \pm 1.46$ & $3.46 \pm 0.37$ \\
ITO/DNA & $15.56 \pm 0.08$ & $0.667 \pm 0.007$ & $51.47 \pm 0.86$ & $5.33 \pm 0.13$ \\
ITO/PEIE & $16.43 \pm 0.10$ & $0.699 \pm 0.014$ & $65.02 \pm 1.27$ & $7.47 \pm 0.26$ \\
ITO/ZnO-NPs & $16.55 \pm 0.10$ & $0.697 \pm 0.004$ & $60.63 \pm 1.16$ & $7.00 \pm 0.13$ \\
ITO/ZnO-NPs/PEIE & $16.79 \pm 0.15$ & $0.723 \pm 0.005$ & $66.47 \pm 0.60$ & $8.07 \pm 0.04$ \\
ITO/DNA/ZnO-NPs & $17.17 \pm 0.14$ & $0.717 \pm 0.010$ & $66.22 \pm 0.74$ & $8.16 \pm 0.17$ \\
ITO/ZnO-NPs/DNA & $18.90 \pm 0.05$ & $0.712 \pm 0.005$ & $61.71 \pm 0.82$ & $8.31 \pm 0.13$ \\
PET_ITO/ZnO-NPs/DNA & $16.92 \pm 0.26$ & $0.716 \pm 0.006$ & $58.29 \pm 0.30$ & $7.06 \pm 0.18$ \\
\hline
\end{tabular}



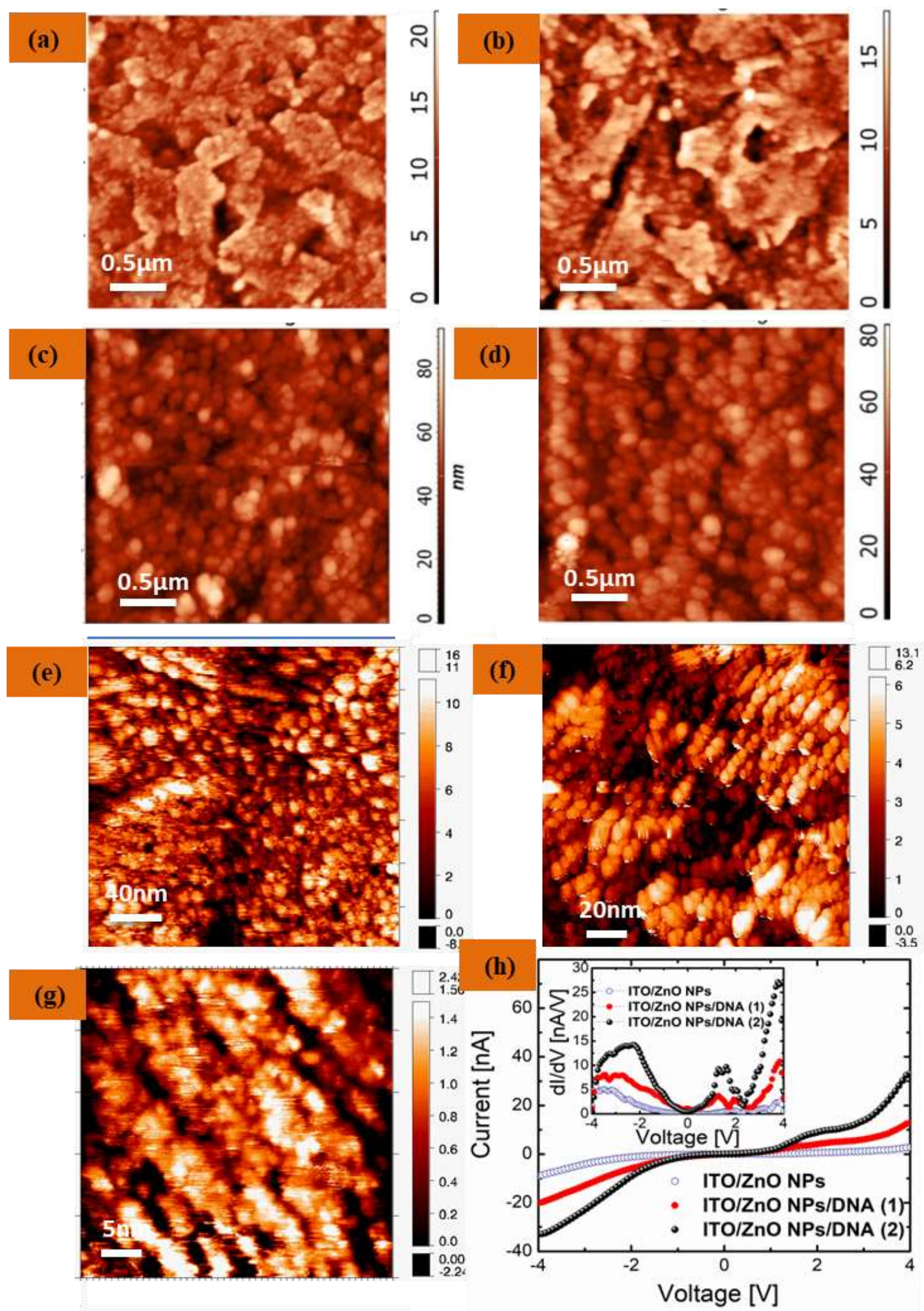
Fig.2 - Atomic Force Microscopy (AFM) images of (a) ITO only substrate; (b) ITO/DNA; (c) ITO/ZnO-NPs and (d) ZnO-NPs coated DNA bilayer (ITO/ZnO-NPs/DNA). The scan size is $2 \mu \mathrm{m}$ $x 2 \mu m$ for all the interlayers. Scanning Tunneling Microscopy (STM) images of (e) ITO/ZnO-NPs surface over a $350 \mathrm{~nm} \times 350 \mathrm{~nm}$ area, (f) ITO/ZnO-NPs surface after DNA deposition over a 400 $\mathrm{nm} \times 400 \mathrm{~nm}$ area; $(\mathrm{g})$ Better-resolved image (50 $\mathrm{nm} \times 50 \mathrm{~nm}$ ), showing the DNA bundles formed on the surface of ZnO-NPs layer. Scanning Tunneling Spectroscopy (STS) response on the ITO/ZnO-NPs before and after DNA deposition; (h) Typical tunneling current vs voltage curves measured on ITO/ZnO-NPs layer (empty hexagon) and after DNA deposition (red (1) and black (2) circles) measured at two different area on the same substrate. Inset: the corresponding $d I / d V$ derivative vs voltage curves.

Atomic Force Microscopy (AFM) and Scanning Tunneling microscopy (STM) measurements were carried out to investigate the surface morphology and roughness of various different interlayers including DNA, ZnO-NPs and ZnO-NPs/DNA over the ITO substrate. As shown in Fig.2(a), the corrugated and typical lamellae surface morphology was observed for bare ITO ("ITO Only") with a root mean square (rms) surface roughness of $2.1 \mathrm{~nm}$. The deposition of DNA does not change the roughness value (Fig.2(b)) since it is very thin (1-6nm) and conformal as measured by $\mathrm{STM}^{11}$. The $\mathrm{ZnO}$ surface consists of a uniform film of nano-sized particles (see Fig. 2(c)) with a rms value of $11 \mathrm{~nm}$. Higher spatially-resolved image of the same surface is provided in Fig.2(e) through STM microscopy, where the $\mathrm{ZnO}$ particle size dimension is about $10 \mathrm{~nm}$. The $\mathrm{ZnO}-\mathrm{NPs}$ surface morphology after DNA deposition through STM, shows more elongated structures, due to the presence of DNA (see Fig.2(e) and 2(f)). Even more elongated structures were visible in our previous study of DNA covering ITO instead of $\mathrm{ZnO}-\mathrm{NPs}^{11}$. The surface roughness of ITO/ZnO- 
NPs/DNA bilayer was $9.3 \mathrm{~nm}$ which is a little lower than that of ZnO-NPs only (11nm). The DNA coverage may thus smoothen in part the surface reducing the exposure of surface defects present on the ZnO-NPs film surface ${ }^{30}$. The STM images (Fig.2(e) and (f) gave a similar trend in the roughness since the rms values are $2.71 \mathrm{~nm}$ and $1.73 \mathrm{~nm}$, respectively and they confirm a global leveling of the ZnO-NPs surface due to the DNA deposition. Fig.2(h), reports the STS (Scanning Tunneling Spectroscopy) response on the ITO/ZnO-NPs substrate before and after DNA deposition. The tunneling current vs voltage curve measured on ITO/ZnO-NPs layer (empty hexagon) shows that the sample has a lower conductance in all the applied voltage range studied. This conductance increases in the same range after DNA deposition (red (1) and black (2) circles). The inset of the figure reports the corresponding $\mathrm{d} / \mathrm{dV}$ derivative vs voltage curves that evidence two prominent features which are completely absent in the $\mathrm{ZnO}-\mathrm{NPs}$ only substrate. The appearance of these contributions indicates that upon DNA deposition a local modification of the energy band structure of the $\mathrm{ZnO}-\mathrm{NPs} / \mathrm{DNA}$ interface took place, as evidenced by the structures located around $-2.5 \mathrm{eV}$ and $+1.8 \mathrm{eV}$ assigned to the electronic states of the $\mathrm{DNA}^{31,32}$. In addition, we note from the symmetry of the $\mathrm{dI} / \mathrm{dV}$ curves with respect of the Fermi level (located at zero voltage of the spectrum), that the interface modification produces a marked increase of the tunneling current and globally favors both the injection and extraction of carriers at the interface. 
An important reason behind the improvement in performance of solar cells with insertion of the additional layer in our composite EELs is the lowering of the overall work function of the electron collecting electrode as occurs also when using other cathodes such as $\mathrm{Ca}$ and $\mathrm{Ba}^{33,34}$.We measured the contact potential difference (CPD) of ITO/DNA, ITO/ZnO-NPs and ITO/ZnO-NPs/DNA using Kelvin probe measurements and compared to that of a clean bare ITO surface as reported in Fig.3(a). The work function was estimated by assigning the value of $4.7 \mathrm{eV}$ to the work function of ITO as determined from previous KP measurements on ITO calibrated utilizing a highly oriented pyrolytic graphite surface ${ }^{35}$. We measured a decrease of the work function of the ITO electrode from $4.7 \mathrm{eV}$ to $4.45 \mathrm{eV}$ after DNA nanolayer incorporation, similarly to what observed when DNA is deposited over a metal such as $\mathrm{Au}, \mathrm{Al} \operatorname{etc}^{15}$ leading to higher rectification and improved cell performance ${ }^{11}$. The lowering of work function is even greater when the $\mathrm{ZnO}-\mathrm{NPs}$ layer is deposited over ITO (from $4.7 \mathrm{eV}$ to $4.3 \mathrm{eV}$ ). Importantly, the work function is lowered further (reaching the lowest value of $4.25 \mathrm{eV}$ ) after deposition of DNA nanolayer over the $\mathrm{ZnO}$ NPs layer (i.e. ITO/ZnO-NPs/DNA). A similar effect also took place when the DNA nanolayer was placed below the $\mathrm{ZnO}-\mathrm{NPs}$ layer (4.27 eV for the $\mathrm{DNA} / \mathrm{ZnO}$ double layer), being lower than $\mathrm{ZnO}$ or DNA only samples although not as low as the ITO/ZnO-NPs/DNA case. We note from Figure $3 \mathrm{~b}$ that the lowering of work function of the ITO/ZnO-NPs/DNA electron collecting electrode matches the values found in the literature for the electron affinity of $\mathrm{PC}_{70} \mathrm{BM}(\sim 4.0-$ $4.3 \mathrm{eV})^{23,36}$ and is furthest away from that of hole transport layer and anode $\left(\mathrm{MoO}_{3} / \mathrm{Ag}\right)$ as displayed in simplified energy band diagram of figure 3 (b)). As a result, the difference generated between energy levels of the hole-collecting $\mathrm{MoO}_{3} / \mathrm{Ag}$ and electron-collecting ITO/ZnONPs/DNA contacts results in an increased built-in potential which plays an important role in charge collection and considerably enhances the $\mathrm{V}_{\mathrm{OC}}$. In fact the $\mathrm{V}_{\mathrm{OC}}=0.71 \mathrm{~V}$ is the highest for the cell 
with the composite cathode confirming the trend found between $V_{O C}$ and work function differences in a systematic study when using more conventional contacts ${ }^{37}$. The lowering of the work function when DNA is used together with $\mathrm{ZnO}-\mathrm{NPs}$ also leads to enhanced tunnelling currents found in STS spectroscopy (see Fig. 2(h)).

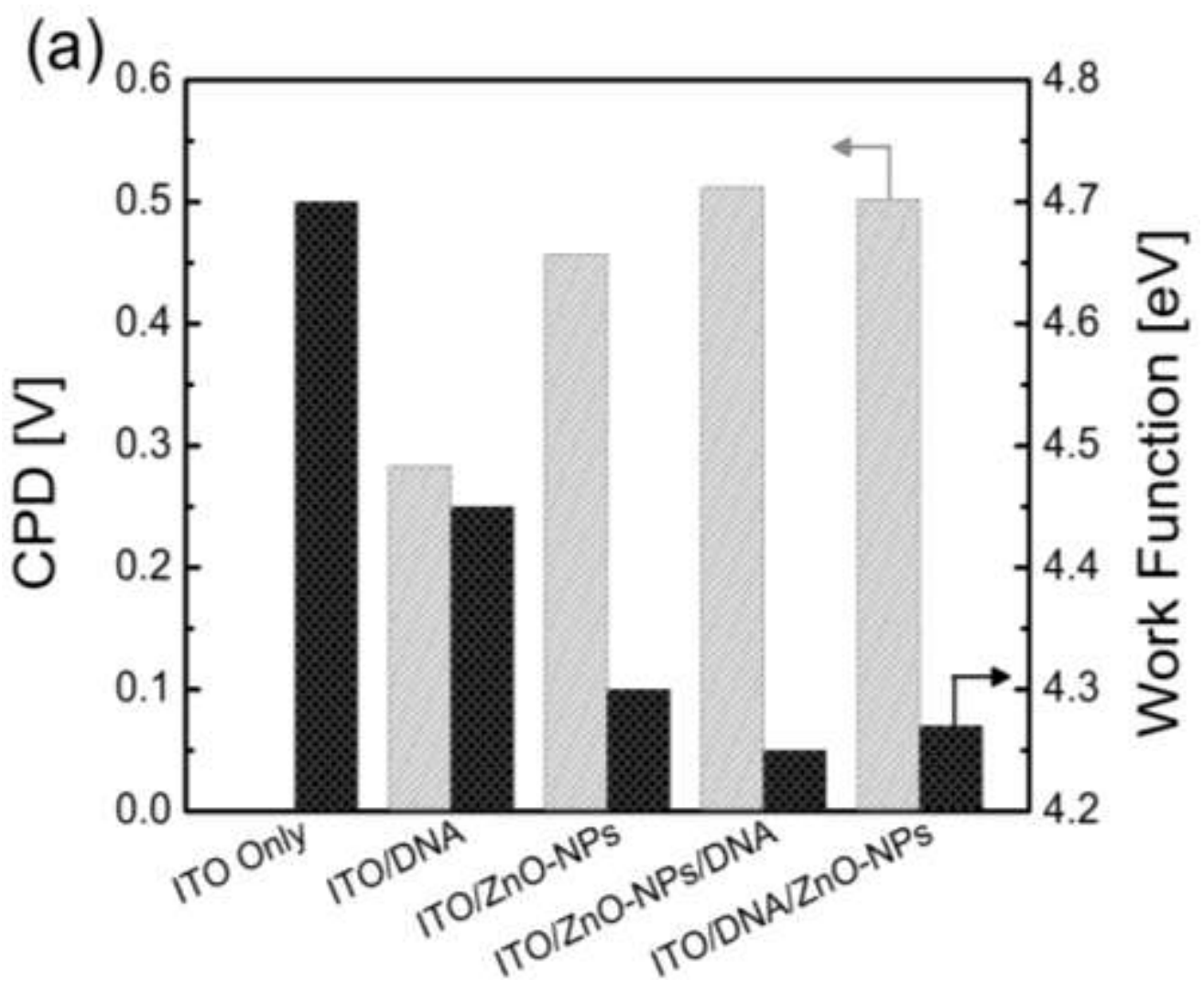


(b)
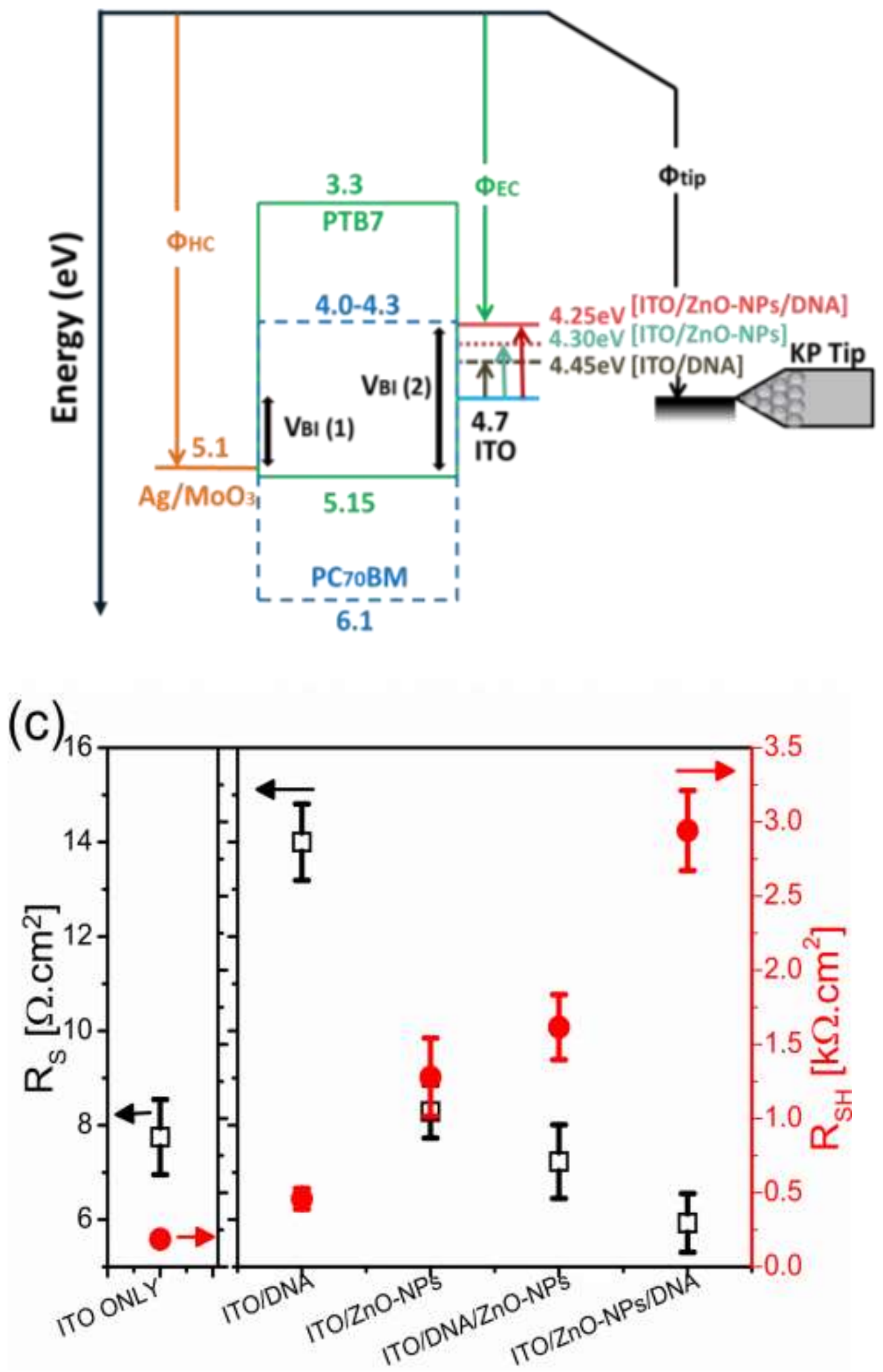
Fig.3 - (a) Work function and CPD for the surface of ITO only, ITO/DNA, ITO/ZnO-NPs and DNA coated ZnO-NPs layer; (b) simplified energy band diagram of PTB7:PC ${ }_{70} B M$ solar cell device with DNA-coated ZnO-NPs bilayer which result in lowering the work function of cathode; (c) Series resistance ( $R_{S}$, open squares) and shunt resistance ( $R_{S H}$, closed circles) extracted from the $J$-V curves of the four cells each under AM1.5G, $1000 \mathrm{~W} / \mathrm{m}^{2}$ irradiation.

The picture described above is supported from analysing dark current density curves of Fig.1(c) where the cells with the electron extracting electrodes with the lowest work functions possess the highest turn-on voltages (e.g. voltages for which the current is $0.1 \mathrm{~mA} / \mathrm{cm}^{2}$ ): significant charge injection in sandwich structures like these only occurs after applying voltages greater than the built in potential which in turn scales with the work function of the contact until pinning occurs ${ }^{38}$. The benefits of the composite EEL also include (see Fig.3(c)) a better series resistance (Rs goes from $8.29 \Omega \mathrm{cm}^{2}$ to $5.93 \Omega \mathrm{cm}^{2}$ ) as well as a significant improvement in shunt resistance ( $\mathrm{R}_{\mathrm{SH}}$ goes from $1.27 \mathrm{~K} \Omega \mathrm{cm}^{2}$ for ZnO-NPs only EELs to $2.94 \mathrm{~K} \Omega \mathrm{cm}^{2}$ for ZnO-NPs/DNA EEL). On the one hand the fact that the Jsc is higher in the $\mathrm{ZnO} / \mathrm{DNA}(18.9 \mathrm{~mA} / \mathrm{cm} 2)$ device compared to the DNA/ZnO device $(17.17 \mathrm{~mA} / \mathrm{cm} 2)$ contributes to the lower FF since Ohmic losses are greater with higher photocurrents. Secondly, Rs and Rsh are calculated at the axes only, and do not follow the shape of the curves at the maximum power points. The contribution of these two factors can explain the lower FF. The short circuit current (Jsc) of the ITO may be higher than the ITO/DNA EEL, even though the built in potential is lower, because of a better contact resistance (and lower Rs as shown in figure $3(\mathrm{c}))$. Thus, not only the bilayer improves carrier extraction but may also provide better hole blocking behaviour ${ }^{14}$ as is also evident in the high rectifying ratios of figure $1 \mathrm{c}$. The positive electron-extracting properties of $\mathrm{ZnO}-\mathrm{NPs}$ stems from an electron affinity that matches well that of the PCBM whilst at the same time guarantees some hole blocking behaviour due to a high 
ionization potential ${ }^{4}$. As discussed previously ${ }^{11}$, that DNA have been attributed mainly to the formation of an interfacial dipole with the phosphate anions in the DNA at its origin ${ }^{39}$. The DNA used in this study is a sodium DNA complex having polar groups $\mathrm{Na}^{+}$which neutralize $\mathrm{PO}_{3}{ }^{-}$ (phosphate anions) ${ }^{16,27}$. The DNA-contained anions can react with the indium and tin ions at the surface of the ITO to form complexes that can lower the work function of such materials ${ }^{11,40,41}$, a likely outcome being able to occur also with $\mathrm{Zn}$. Thus, the $\mathrm{ZnO}-\mathrm{NPs} / \mathrm{DNA}$ double layer leads to better matching of the electron-conducting levels of the EEL with that of PCBM compared to the neat counterparts. A better coverage may also ensure blocking behaviour as described previously. Apart from the change in work function and in possible blocking behavior of the differing EELs affecting the performance of the cells, one needs to consider that some morphological change may also occur. In fact, the morphology of the $\mathrm{PTB} 7: \mathrm{PC}_{71} \mathrm{BM}$ blend not only depends on different solvents and additives of the blend ink ${ }^{42}$ but also on the surface onto which the films are deposited. For example, the morphology of the $\mathrm{PTB} 7: \mathrm{PC}_{70} \mathrm{BM}$ blend can differ to different degrees when PEIE is deposited on top of bare $\mathrm{ZnO}-\mathrm{NPs}^{30,43}$. Although to what extent each contribution affects photovoltaic performance is difficult to quantify, the effect of the EELs on order can be focus of future investigations ${ }^{44}$. Note that the bilayer based solar devices also delivered an improvement in the stability of un-encapsulated cells left in air (see shelf life plots in Fig S3). We calculated that PCE decrease was $\sim 41 \%$ for the $\mathrm{ZnO}-\mathrm{NPs} / \mathrm{DNA}$ solar cells after 30 days in air whereas it was $62 \%$ lower for the ZnO-NPs cells. The improvement in stability of $\mathrm{ZnO}-\mathrm{NPs} / \mathrm{DNA}$ based solar cells is due to a smaller lowering of FF and Voc compared to those of ZnO-NPs based devices. Although we do not have other correlated experiments, the evidence points to the composite layers retaining their extracting/blocking properties more effectively in time. 
(a)
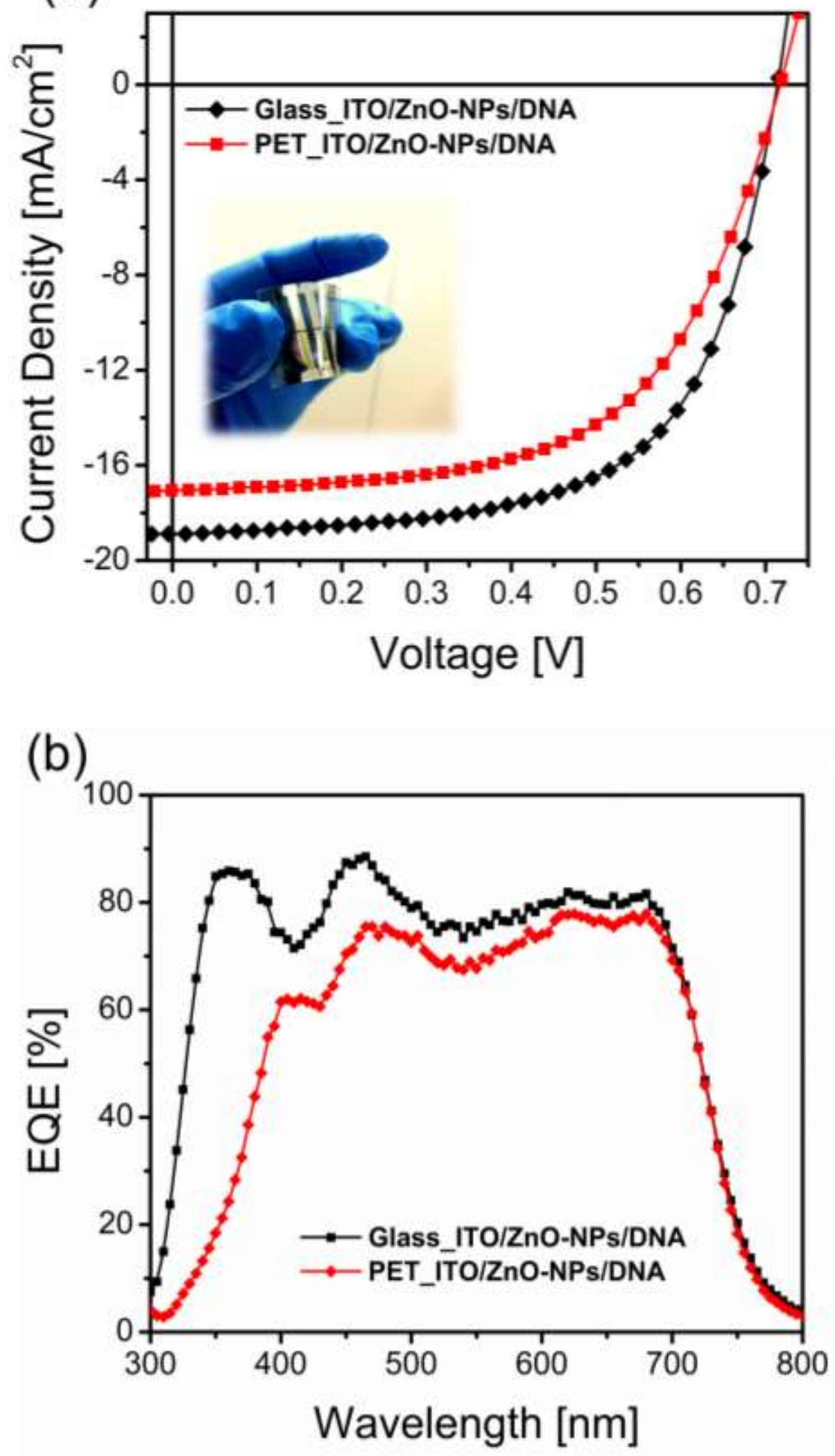
Fig.4 - (a) Best current density-voltage curves of ITO/ZnO-NPs/DNA/PTB7:PC ${ }_{70} B M / M o O_{3} / \mathrm{Ag}$ solar cell devices fabricated on glass (black solid diamond) and PET substrates red solid square under AM1.5G, $1000 \mathrm{~W} / \mathrm{m}^{2}$ irradiation; (b) EQE spectra of ITO/ZnONPs/DNA/PTB7:PC $70 B M / M o O_{3} / A g$ inverted solar cell on glass and PET substrates.

We also fabricated a set of devices on flexible PET substrates. The $J$ - $V$ characteristics of the best cell for inverted PTB7:PC $70 \mathrm{BM}$ solar cells with the composite $\mathrm{ZnO-NPs/DNA} \mathrm{bilayer} \mathrm{EEL} \mathrm{on}$ PET/ITO is shown in Fig.4(a) together with the curves of the same cell architecture on glass. The average values of PV parameters of DNA coated ZnO-NPs bilayer on both glass and flexible substrates were compared and summarized in table1 and Fig. S1. The PCE of the best flexible cells was 7.1\%. The EQE spectra of the solar cell devices with both glass and PET substrates are shown in Fig.4(b) The EQE value for flexible substrate based device peaks at $\sim 75 \%$ at around $450 \mathrm{~nm}$ wavelength, not as high as the glass counterparts, furthermore the EQE was reduced significantly below 400nm where the PET absorbs more than its glass counterpart ${ }^{45}$.

In conclusion, the performance of $\mathrm{PTB} 7: \mathrm{PC}_{70} \mathrm{BM}$ inverted solar cells was improved after successfully incorporating a composite $\mathrm{ZnO}-\mathrm{NPs} / \mathrm{DNA}$ electron-extracting bilayer between the ITO bottom contact and the blend photoactive layer. It led to a lowering of the work function compared to ITO/DNA and ITO/ZnO-NPs only layer as measured by Kelvin probe. The surface morphology measurements (AFM and STM) also show reduction in surface roughness of ITO/ZnO-NPs layer after the deposition of the DNA nanolayer. The ITO/ZnO-NPs/DNA also leads to the highest shunt resistances as well as rectification ratios. As a result, the power conversion efficiency of the cells was the highest, i.e. a maximum PCE of $8.5 \%$ which 
corresponded to a $\sim 19 \%$ increment compared to solar cells with a ZnO-NPs EEL layer only. This is the first instance ever reported where any transport layer incorporating biological material (in our case DNA which is a naturally occurring premium biological genetic material) reaches state of the art efficiencies which are of real practical interest $(\mathrm{PCE}>8 \%)$ thus elevating transport layers with biological matter to the level of synthetic ones. We also report that ZnO/DNA bilayer leads to even better performance (improvement of 5\% in power conversion efficiency, PCE, in relative terms) compared to the most efficient composite EELs (ZnO/PEIE) for polymer solar cells. Furthermore, DNA over $\mathrm{ZnO}$ does not need any further annealing treatment compared to other EEL such as PEIE which are annealed at temperatures reaching up to $150{ }^{\circ} \mathrm{C}$. This has allowed us to successfully apply the whole process to flexible substrates, obtaining an efficiency of $7.2 \%$. Furthermore, the casting from water/alcohol solvents also suggests a convenient and ecofriendly processes for the formation of very efficient electron extraction layers. We have also shown that DNA/ZnO bilayer leads to better air stability compared to just ZnO-NPs layer based solar cell devices. In the future, DNA can be further functionalized with targeted molecular groups or ions over the $\mathrm{ZnO}$ layer that can improve the performance of the device further and pave the way for future studies and concepts in polymer-based optoelectronic devices such as bio-detectors and medical sensors, an expanding field with great potential for the future.

\section{Experimental}

Materials: DNA (Deoxyribonucleic acid sodium salt from salmon testes, molecular mass of $1.3 \times$ $10^{6} \mathrm{Da}(\sim 2,000$ base pair)) was purchased from Sigma Aldrich. Zinc oxide, dispersion nanoparticles (ZnO-NPs) of the average particle size less than 40nm and with 20 wt.\% concentration in water was also purchased from Sigma Aldrich. PTB7, Poly[[4,8-bis[(2- 
ethylhexyl)oxy]benzo[1,2-b:4,5-b']dithiophene-2,6-diyl][3-fluoro-2-[(2

ethylhexyl)carbonyl]thieno[3,4-b]thiophenediyl]] and $\mathrm{PC}_{70} \mathrm{BM}$, [6,6]-Phenyl-C71-butyric acid methyl ester, (99.99\%) and $\mathrm{PC}_{60} \mathrm{BM}$ were purchased from Solarmer and Solenne BV, respectively. Molybdenum oxide (MoO3, 99.98\% powder), ortho-Xylene and silver (Ag, wire Z 99.99\%) were purchased from Sigma Aldrich.

Device Fabrication: In the process of inverted solar cell device fabrication, at first ITO glasscovered substrates (Kintec $-8 \Omega / \square$ ), patterned with wet-etching in hydrobromic acid (HBr) and cleaned in ultrasonic bath in acetone and isopropanol for 10min with each solvent. For the fabrication of ITO/ZnO NPs/DNA/ PTB7:PC ${ }_{70} \mathrm{BM} / \mathrm{MoO}_{3} / \mathrm{Ag}$ solar cells, at first ZnO-NPs solution for the thin film deposition was prepared by diluting $200 \mu 1 \mathrm{ZnO}-\mathrm{NPs}$ dispersion in $20 \mathrm{ml}$ ethanol in air which was further stirred overnight at room temperature. ZnO-NPs thin film was deposited on ITO coated glass/PET substrate by spin-coating at spin speed of $2500 \mathrm{rpm}$ which was carried out and then annealed at $140{ }^{\circ} \mathrm{C}$ for 20 minutes in air. The DNA solution was prepared first by dissolving $5 \mathrm{mg}$ DNA fibers in $0.5 \mathrm{ml}$ of deionized water until the solution becomes transparent and then $4.5 \mathrm{ml}$ of methanol was added to dilute it to be $1 \mathrm{mg} / \mathrm{ml}$ concentration. After overnight stirring at room temperature, the DNA solution was filtered using a $0.2 \mu \mathrm{m}$ PVDF filter (NB: it is important to use this type of filter to obtain the films discussed in this article; the use of PTFE or polypropylene filters with the same pore size instead, resulted in DNA films with different optical and thickness properties) and then spin-coated on ITO coated glass substrate at spin speed of $3000 \mathrm{rpm}$ over the ZnO-NPs thin film in air. The prepared ITO/ZnO-NPs/DNA samples were left in vacuum overnight for drying (we note from Kelvin probe measurements that work function of the electrodes changes if these samples are left in air so drying/inert atmosphere fabrication is important) after that samples were directly transferred in inert gas atmosphere. From AFM and 
transmittance measurements (not shown), it is clear that both layers (ZnO-NPs and DNA) are present after deposition of both layers even if both are soluble in the similar solvent. The active layer of polymer contents PTB7:PC $70 \mathrm{BM}(1: 1.5)$ dissolved in ortho-xylene and 3\%v/v of 1,8diiodooctane (DIO) was spin coated in inert gas atmosphere first at 500rpm for 80 seconds, leading further to $1500 \mathrm{rpm}$ for next 10 seconds for obtaining $80 \mathrm{~nm}$ thickness which was confirmed by profilometer (Dektak 150). The coated films were kept in vacuum for 20 minutes drying. At the end all substrates were transferred in the metal evaporator where $\mathrm{MoO}_{3}$ (hole extracting interlayer)

and silver top contact were thermally evaporated through a shadow mask at pressures below $10^{-6}$ mbar for a final thickness of $5 \mathrm{~nm}$ and $100 \mathrm{~nm}$ respectively ${ }^{8}$. Each substrate contained 4 devices of $0.1 \mathrm{~cm}^{2}$ area.

\section{Characterization and Measurements:}

Atomic Force Microscopy: The surface morphology of all interlayers (DNA, ZnO-NPs and DNA coated $\mathrm{ZnO}-\mathrm{NPs}$ bilayer) along ITO only were measured in inert atmosphere using AFM technique. All samples were prepared by spin coating over the pre-cleaned ITO substrates. AFM images have been obtained with a commercial AFM (NTMDT NTEGRA) in tapping mode with cantilever frequency of 140-390kHz (NSG10).

Kelvin Probe Measurements: The conduction band energy levels (WFs) of the bare ITO electrode and after deposition of various different interfacial layers including DNA, ZnO-NPs and DNA coated $\mathrm{ZnO}-\mathrm{NPs}$ bilayer were measured using Kelvin probe microscopy in inert atmosphere. KPFM measurements in this study have been performed using the AFM NTMDT operating in amplitude detection mode with a Vpp applied of $100 \mathrm{mV}$. A cantilever with a conductive TiN tip 
(calibrated with HOPG) (NSG01/TiN) have been used. The compensation voltage needed to nullify the $\mathrm{AC}$ voltage generates the Contact potential map (CP). The average $\mathrm{CP}$ have been then calculated for each surface scan. For each sample, five different areas have been scanned and a mean of these five CP's values have been calculated as representative of the final sample CP. Note that great care was taken in ensuring transport and measurements of the samples was carried out in inert nitrogen atmosphere. When KP measurements were instead performed in air, the readings led to significantly higher values of the work function, especially for the DNA samples, due to the hygroscopic nature of the surface (and must thus be avoided).

Scanning Tunneling Spectroscopy: The STM measurements were carried out under ultra-high vacuum conditions (base pressure $\sim 4 \times 10^{-10} \mathrm{mbar}$ ) at room temperature (by using an Omicron system), using etched W (tungsten) tip tips. The samples were inserted in the UHV chamber immediately just after their preparation to prevent from the contamination and degradation due to air exposure. The topography images reported were acquired in constant current mode and the images presented were obtained from unfiltered data apart from rigid plane subtraction. Scanning tunneling spectroscopy (STS) data were acquired with the same the apparatus. In this operation mode the tunneling current was registered as a function of the applied bias (I vs V) while acquiring the topography images. The feedback loop was disabled for short time and the set-point current, which regulated the tip-sample distance, remained unchanged during the voltage scan. The I-V curves were collected over grids of points equally spaced on the scanned sample area and the spectra reported were averaged over a set of several curves. 
Device Measurements: Solar cell electrical characteristics were measured in air with a Keithley2420 source meter under an AM1.5G Class A ABET solar simulator at an intensity of $1000 \mathrm{~W} / \mathrm{m}^{2}$ calibrated with an ECO Pyranometer MS-602 at room temperature. The voltage step, scan speed and delay time for data point scans were fixed at $20 \mathrm{mV}, 1 \mathrm{~s}$, and $200 \mathrm{~ms}$ respectively. Forward and reverse sweeps gave negligible hysteresis. Cells were masked with $0.1 \mathrm{~cm}^{2}$ black tapes during measurements. The EQE measurements were performed in air using IPCE (Incident Photon-to-current Conversion Efficiency) system (IPCE-LS200, Dyers) which has been calibrated using UV-enhanced Si detector (Thorlabs, 250-1100nm).

\section{Supporting Information}

Average data plots of different interlayer based $\mathrm{PTB} 7 / \mathrm{PC}_{70} \mathrm{BM}$ solar cell devices together with EQE spectra. The stability measurements of the best solar cell devices. Summarized data table of all electron extraction/transport layer based solar cell devices.

\section{Acknowledgments}

We thank Dario Di Carlo Rasi, Dr.Andrea Zampetti, Matteo Gasbarri, Sergio Alexis Castro Hermosa, Dr Luca La Notte, Dr Giampaolo Susanna, Dr Francesca Brunetti, Prof Andrea Reale and Prof Aldo Di Carlo for useful discussions. We thank MIUR for PRIN 2012 (2012A4Z2RY) "AQUASOL" (Celle solari polimeriche processabili da mezzi acquosi: dai materiali ai moduli fotovoltaici) and the EU CHEETAH project for financial support.

\section{References}

1. G. Yu, J. Gao, J. C. Hummelen, F. Wudl and A. J. Heeger, Science, 1995, 270, 1789-1791. 
2. C. J. Brabec, S. Gowrisanker, J. J. Halls, D. Laird, S. Jia and S. P. Williams, Advanced materials, 2010, 22, 3839-3856.

3. M. Granstrom, K. Petritsch, A. C. Arias, A. Lux, M. R. Andersson and R. H. Friend, Nature, 1998, 395, 257-260.

4. C. Han, Y. Cheng, L. Chen, L. Qian, Z. Yang, W. Xue, T. Zhang, Y. Yang and W. Cao, ACS Applied Materials \& Interfaces, 2016, 8, 3301-3307.

5. S. Cho, K.-D. Kim, J. Heo, J. Y. Lee, G. Cha, B. Y. Seo, Y. D. Kim, Y. S. Kim, S.-y. Choi and D. C. Lim, Scientific Reports, 2014, 4, 4306.

6. S. Woo, W. Hyun Kim, H. Kim, Y. Yi, H.-K. Lyu and Y. Kim, Advanced Energy Materials, 2014, 4, 1301692-n/a.

7. H.-L. Yip and A. K.-Y. Jen, Energy \& Environmental Science, 2012, 5, 5994-6011.

8. G. Susanna, L. Salamandra, C. Ciceroni, F. Mura, T. M. Brown, A. Reale, M. Rossi, A. Di Carlo and F. Brunetti, Solar Energy Materials and Solar Cells, 2015, 134, 194-198.

9. M. A. Green, A. Ho-Baillie and H. J. Snaith, Nat Photon, 2014, 8, 506-514.

10. P. Ensslen, S. Gartner, K. Glaser, A. Colsmann and H. A. Wagenknecht, Angewandte Chemie (International ed. in English), 2016, 55, 1904-1908.

11. J. Dagar, M. Scarselli, M. De Crescenzi and T. M. Brown, ACS Energy Letters, 2016, 1, $510-515$.

12. A. R. b. M. Yusoff, J. Kim, J. Jang and M. K. Nazeeruddin, ChemSusChem, 2016, 9, 17361742.

13. J. A. Hagen, W. Li, A. J. Steckl and J. G. Grote, Applied Physics Letters, 2006, 88, 171109.

14. P. Zalar, D. Kamkar, R. Naik, F. Ouchen, J. G. Grote, G. C. Bazan and T.-Q. Nguyen, Journal of the American Chemical Society, 2011, 133, 11010-11013. 
15. Y. Zhang, M. Wang, S. D. Collins, H. Zhou, H. Phan, C. Proctor, A. Mikhailovsky, F. Wudl and T.-Q. Nguyen, Angewandte Chemie International Edition, 2014, 53, 244-249.

16. Y. Zhang, P. Zalar, C. Kim, S. Collins, G. C. Bazan and T.-Q. Nguyen, Advanced materials, 2012, 24, 4255-4260.

17. L.-M. Chen, Z. Hong, G. Li and Y. Yang, Advanced materials, 2009, 21, 1434-1449.

18. S. Sakohara, M. Ishida and M. A. Anderson, The Journal of Physical Chemistry B, 1998, 102, 10169-10175.

19. Z. He, C. Zhong, S. Su, M. Xu, H. Wu and Y. Cao, Nat Photon, 2012, 6, 591-595.

20. A. Punzi, F. Nicoletta, G. Marzano, C. G. Fortuna, J. Dagar, T. M. Brown and G. M. Farinola, European Journal of Organic Chemistry, 2016, 2016, 3233-3242.

21. J. Dagar, P. Tyagi, R. Ahmad, R. Singh, O. P. Sinha, C. K. Suman and R. Srivastava, RSC Advances, 2015, 5, 8397-8403.

22. D. Liu and T. L. Kelly, Nat Photon, 2014, 8, 133-138.

23. A. K. Kyaw, D. H. Wang, V. Gupta, J. Zhang, S. Chand, G. C. Bazan and A. J. Heeger, Advanced materials, 2013, 25, 2397-2402.

24. W. J. E. Beek, M. M. Wienk and R. A. J. Janssen, Advanced materials, 2004, 16, 10091013.

25. Y. W. Kwon, C. H. Lee, D. H. Choi and J. I. Jin, Journal of Materials Chemistry, 2009, 19, 1353-1380.

26. T. B. Singh, N. S. Sariciftci and J. G. Grote, Organic Electronics, 2009, 73-112.

27. S. Zamenhof, Methods in Enzymology, 1957, 3, 696-704.

28. L. Wang, J. Yoshida, N. Ogata, S. Sasaki and T. Kajiyama, Chemistry of Materials, 2001, 13, 1273-1281. 
29. G. Zhang, L. Wang, J. Yoshida and N. Ogata, Asia-Pacific Optical and Wireless Communications Conference and Exhibit, 2001, 4580, 337-346.

30. P. Li, L. Cai, G. Wang, D. C. Zhou, J. Xiang, Y. J. Zhang, B. F. Ding, K. Alameh and Q. L. Song, Synthetic Metals, 2015, 203, 243-248.

31. M. Tsuburaya, H. Sakamoto and K. Mizoguchi, Physical Review E, 2014, 89, 022719.

32. R. G. Endres, D. L. Cox and R. R. P. Singh, Reviews of Modern Physics, 2004, 76, 195214.

33. V. Gupta, A. K. K. Kyaw, D. H. Wang, S. Chand, G. C. Bazan and A. J. Heeger, Scientific reports, 2013, 3, 1965.

34. A. K. K. Kyaw, D. H. Wang, D. Wynands, J. Zhang, T.-Q. Nguyen, G. C. Bazan and A. J. Heeger, Nano Letters, 2013, 13, 3796-3801.

35. A. Zampetti, F. D. Rossi, F. Brunetti, A. Reale, A. D. Carlo and T. M. Brown, Applied Physics Letters, 2014, 105, 063304.

36. Y. Yang, W. Chen, L. Dou, W.-H. Chang, H.-S. Duan, B. Bob, G. Li and Y. Yang, Nat Photon, 2015, 9, 190-198.

37. A. Zampetti, A. H. Fallahpour, M. Dianetti, L. Salamandra, F. Santoni, A. Gagliardi, M. Auf der Maur, F. Brunetti, A. Reale, T. M. Brown and A. Di Carlo, Journal of Polymer Science Part B: Polymer Physics, 2015, 53, 690-699.

38. T. M. Brown, R. H. Friend, I. S. Millard, D. J. Lacey, T. Butler, J. H. Burroughes and F. Cacialli, Journal of Applied Physics, 2003, 93, 6159-6172.

39. Y. Zhang, P. Zalar, C. Kim, S. Collins, G. C. Bazan and T. Q. Nguyen, Advanced materials, 2012, 24, 4255-4260.

40. G. E. Plum and K. J. Breslauer, Journal of molecular biology, 1995, 248, 679-695. 
41. K. Sun, H. Zhang and J. Ouyang, Journal of Materials Chemistry, 2011, 21, 18339-18346.

42. S. Guo, W. Wang, E. M. Herzig, A. Naumann, G. Tainter, J. Perlich and P. MüllerBuschbaum, ACS Applied Materials \& Interfaces, 2017, 9, 3740-3748.

43. S. Nam, J. Seo, S. Woo, W. H. Kim, H. Kim, D. D. C. Bradley and Y. Kim, Nature Communications, 2015, 6, 8929.

44. F. Toschi, D. Catone, P. O'Keeffe, A. Paladini, J. Dagar, and T. M. Brown, unpublished results.

45. V. Zardetto, T. M. Brown, A. Reale and A. Di Carlo, Journal of Polymer Science Part B: Polymer Physics, 2011, 49, 638-648. 